\title{
TRADE POLICY RESPONSES TO THE CONTEMPORARY CHALLENGES OF THE GLOBAL MARKET
}

\section{LELA JAMAGIDZE}

\author{
Phd, Assistant professor
}

Ivane Javakhishvili Tbilisi State University, Georgia

lela.jamagidze@tsu.ge

Abstract. The present paper discusses several new trends in the global trade such as digitalization of trade, regional trade agreements and the emergence of new global players. Based on theoretical research and the study of the available secondary statistical data it analyzes how trade policy can address these changes under the existing world trading system.

Trade rules underlying the traditional trading system are still applicable under increasing digital trade and enhanced bilateral and regional trade integration. However, WTO Agreement has nothing to do with those barriers of trade that go beyond traditional trade measures and are very acute for the developing countries. They involve access to the Internet and other telecommunication infrastructure, weak formal institutions and legal protection, lack of awareness about the potential benefits and opportunities in digital trade and lack of IT literacy. In order to overcome these barriers developing countries should incorporate trade policy as a component of deep economic reforms. The pace of development of the multilateral trading rules is slower than required by increasingly flexible business models. Therefore, countries try to find solutions at the bilateral and plurilateral levels. For instance, the EU develops regulations under Digital Single Market as well as within its bilateral trade agreements with non-member states. Georgia is implementing digital economy regulations in accordance to its DCFTA with the EU.

Access to information and communication technologies is essential to be engaged in digital trade. For developing countries investments in digital infrastructure is an important policy issue, while developed countries are more focused on balanced trade rules, which ensure the development of digital trade, on the one hand and security and data protection, on the other.

Contemporary trade policy goes beyond regulation based on classical trade instruments. It encompasses regulation within regional trade and investment agreements, technical standards and other behind-the-border measures, regulation of services and intellectual property markets and support for sustainable development goals, etc. A large part of the contemporary regional trade agreements covers all these areas. The development of bilateral and regional cooperation by encouraging deep integration can be discussed as an effort to overcome inflexibilities of the multilateral system. Regional integration enables countries to develop selective approach towards their trade partners and maintain certain degree of autonomy by applying different trade rules towards different partners. Elimination of behind the border barriers and enhanced regulatory harmonization leads to reduced trade costs, but it also leads to race to the bottom in regulatory facilitation what might be detrimental to the national policy goals. The effects of deep economic integration cannot be assessed based on the traditional approach of trade creation and trade diversion, because integration as an institutional process affects not only trade flows but also economic and institutional development levels of the countries.

Deep regional integration reduces institutional differences within regions and increases them across regions. Besides it, the increasing role of BRICs countries in international trade leads to greater diversity of actors in the world market. New global players ask for greater voice in the process of reforming trade rules. Therefore, global trade rules should reflect the diversity that comes from changes in the patterns as well as the main players of the world market.

KEYWORDS: TRADE POLICY, DIGITAL TRADE, REGIONAL TRADE AGREEMENTS, TRADE RULES.

For citation: Jamagidze, L. (2020). Trade Policy Responses to the Contemporary Challenges of the Global Market. Globalization and Business, №9, pp. 70-76. (In Georgian) https://doi.org/10.35945/gb.2020.09.008 


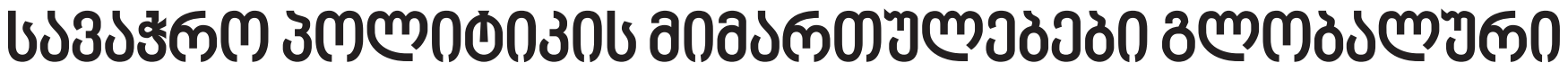

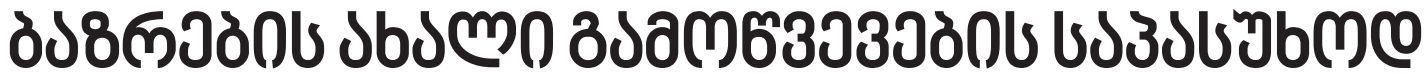

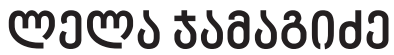

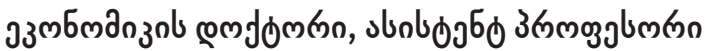

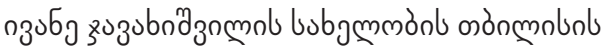

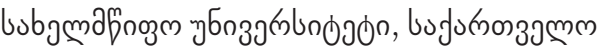

lela.jamagidze@tsu.ge

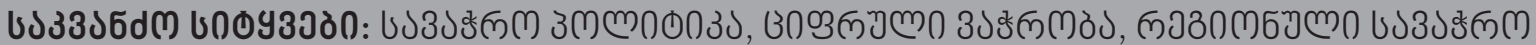

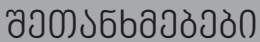

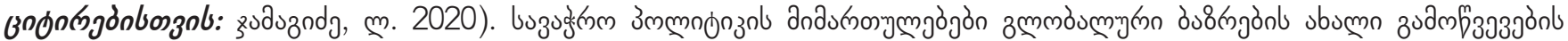

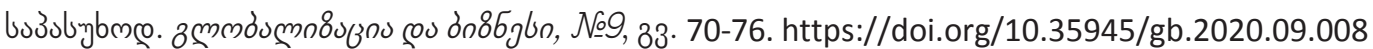

\section{ฆอ৮১З১ल0}

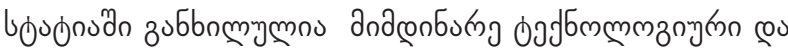

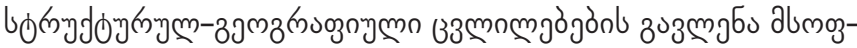

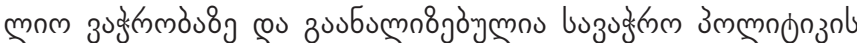

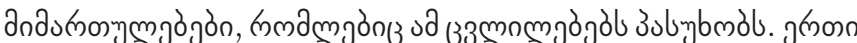

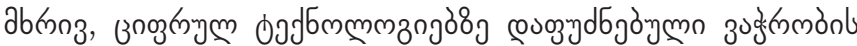

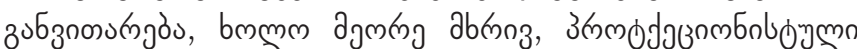

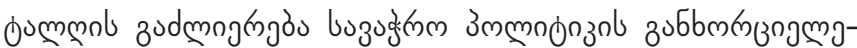

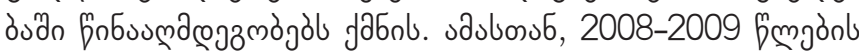

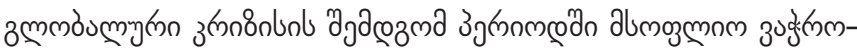

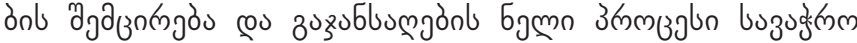

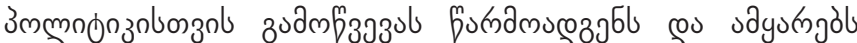

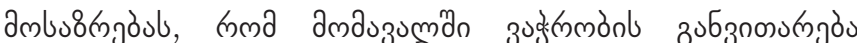

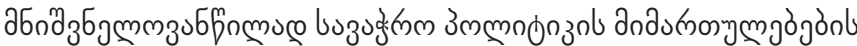

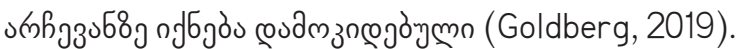

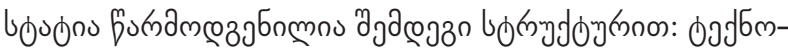

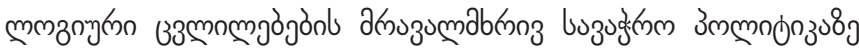

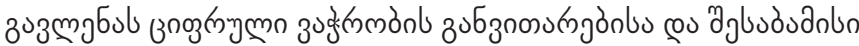

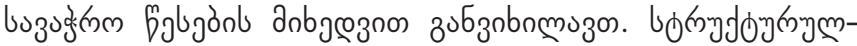

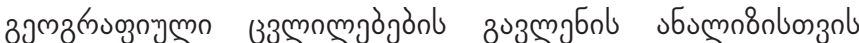

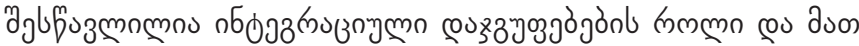

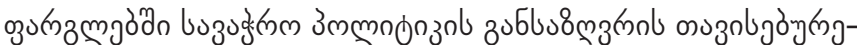

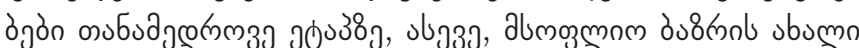

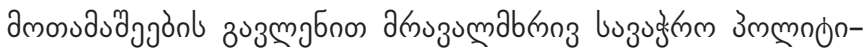

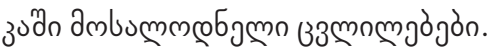

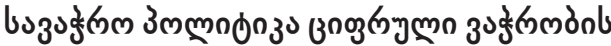

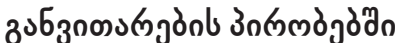

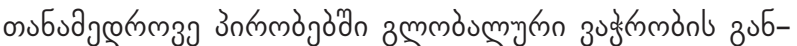

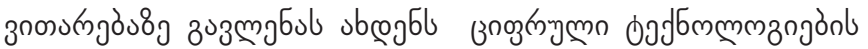

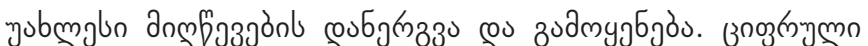

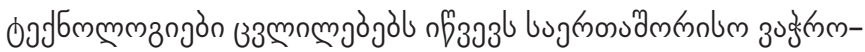

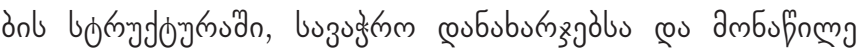

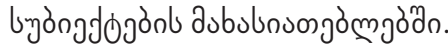

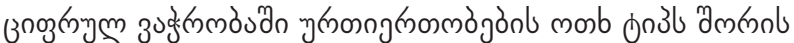

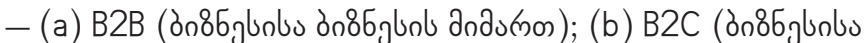

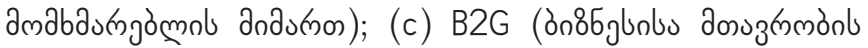
andu(no) co (d) C2C (amabaungàmolus amabaumadomols

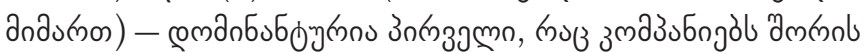

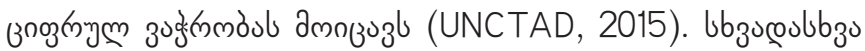

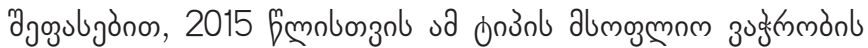

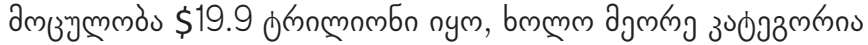

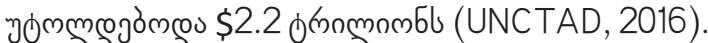

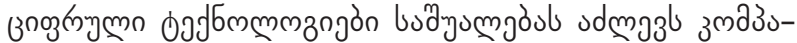

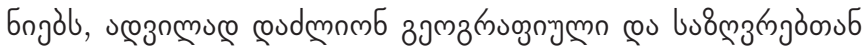
cos zu3

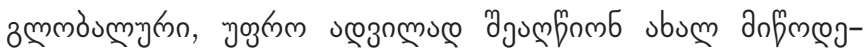

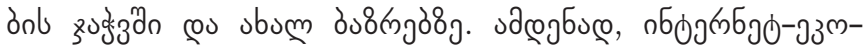

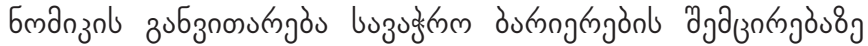

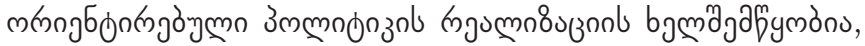

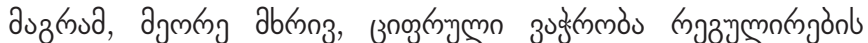
озимmbs8

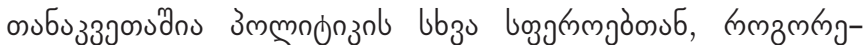

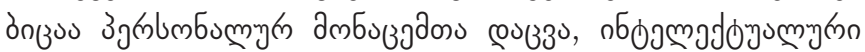

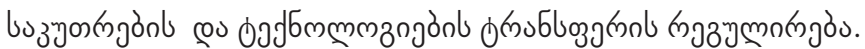

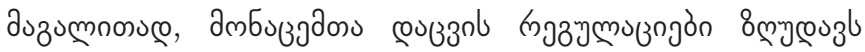

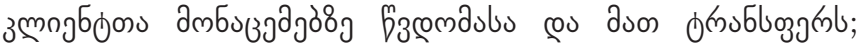

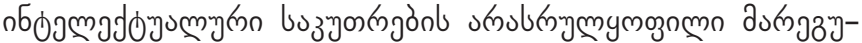

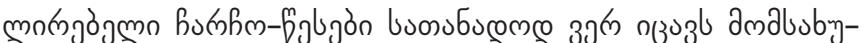

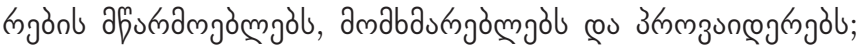

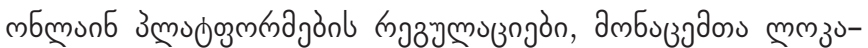

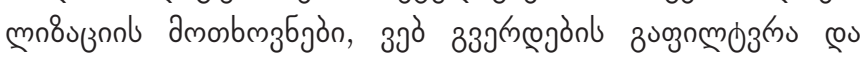




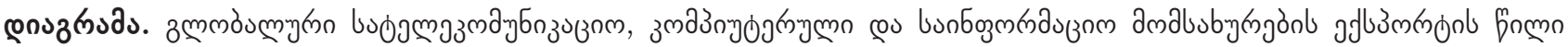

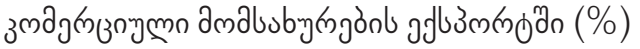

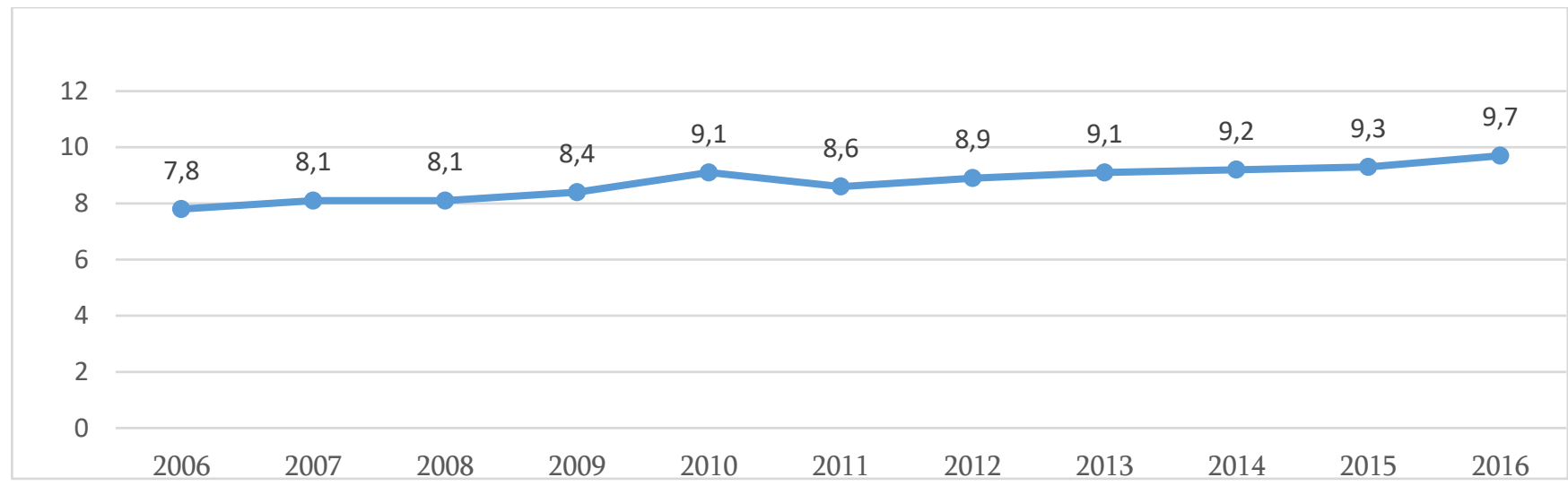

fyußrm: UNCTAD, 2017

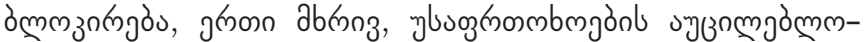

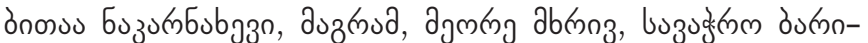

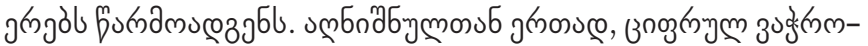

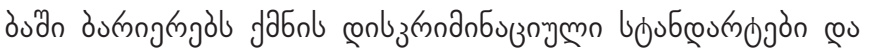
оjbon

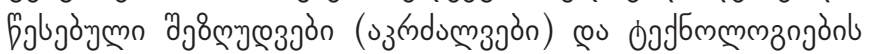

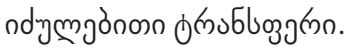

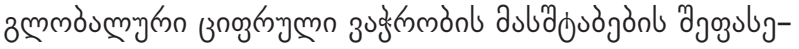

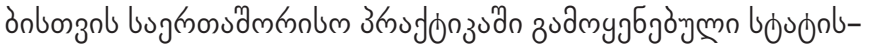

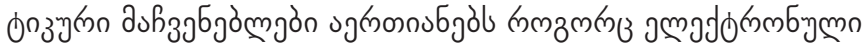

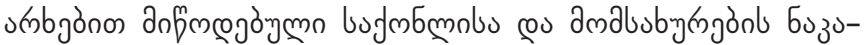

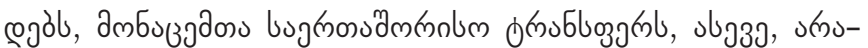

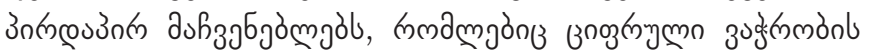

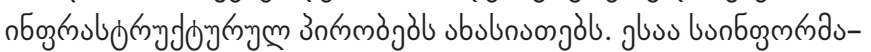

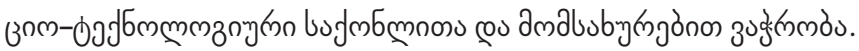

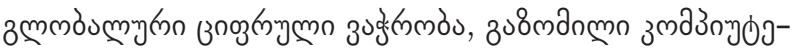

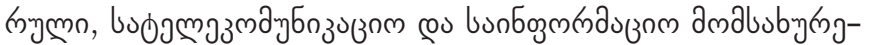

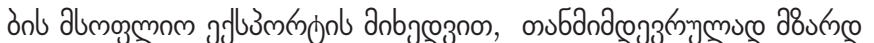

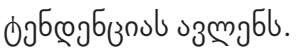

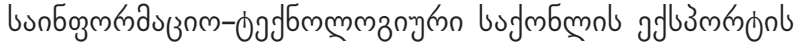

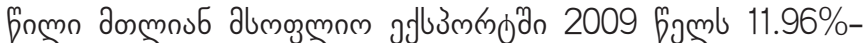

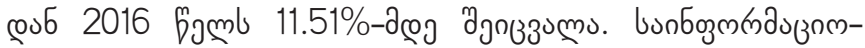

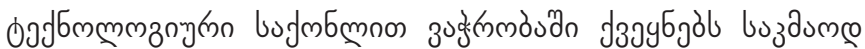

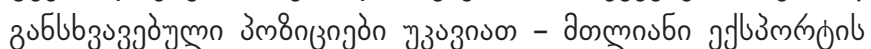

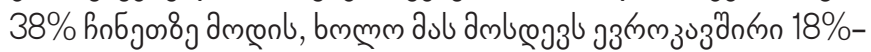

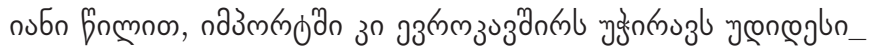

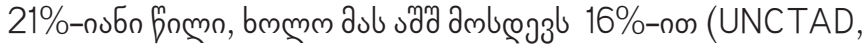

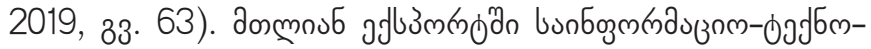

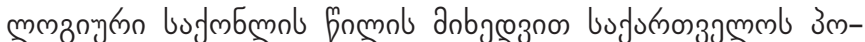

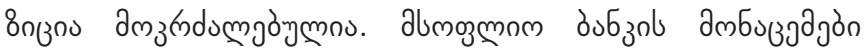

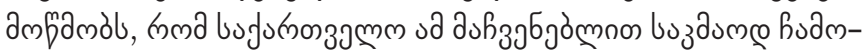

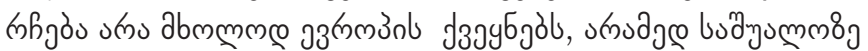

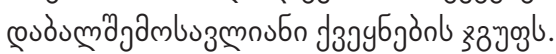

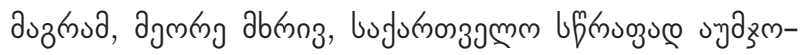

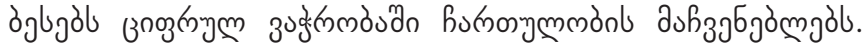

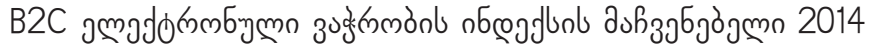

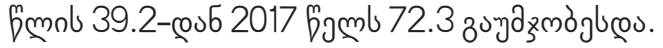

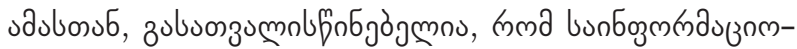

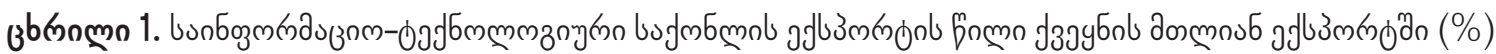

\begin{tabular}{|c|c|c|c|c|c|c|c|c|c|}
\hline Ј3эуงбs & 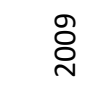 & 올 & $\underset{\sim}{\stackrel{7}{2}}$ & 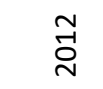 & $\stackrel{m}{\stackrel{m}{\sim}}$ & 节 & $\stackrel{n}{\stackrel{n}{\sim}}$ & 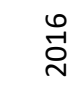 & 今े \\
\hline 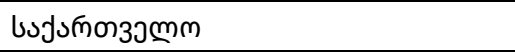 & 0.28 & 0.28 & 0.30 & 0.46 & 0.69 & 0.63 & 1.05 & 0.45 & 0.56 \\
\hline эзп்тззздппп & 6.20 & 6.34 & 5.74 & 5.27 & 4.99 & 5.12 & 5.28 & 5.32 & 5.25 \\
\hline 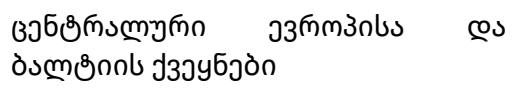 & 13.08 & 13.16 & 11.56 & 10.55 & 9.81 & 9.77 & 9.80 & 9.29 & 9.20 \\
\hline 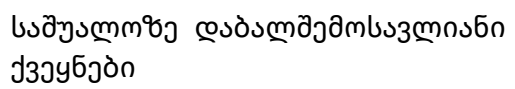 & 5.15 & 4.13 & 4.03 & 4.94 & 5.26 & 5.74 & 7.27 & 7.91 & 3.93 \\
\hline
\end{tabular}

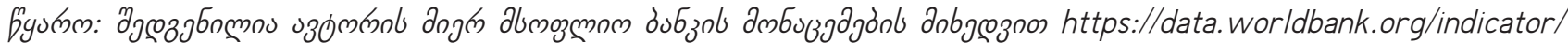
$T X . V A L . I C T G . Z S . U N$ ?contextual=region\&locations $=G E$ 


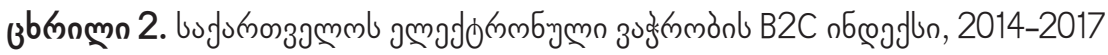

\begin{tabular}{|c|c|c|c|c|}
\hline & 2014 & 2015 & 2016 & 2017 \\
\hline 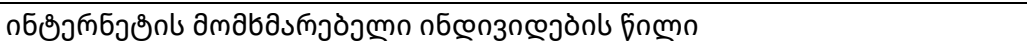 & 49 & - & 50 & 60 \\
\hline 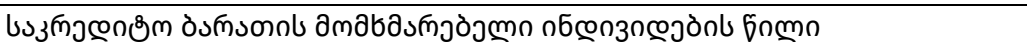 & 18 & 40 & 40 & 61 \\
\hline 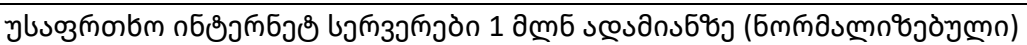 & 62 & 62 & 60 & 72 \\
\hline 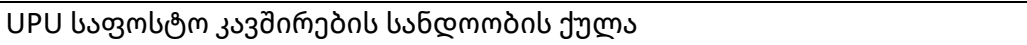 & 28 & 28 & 76 & 96 \\
\hline 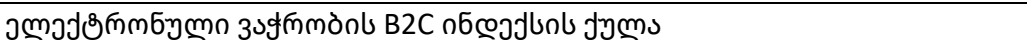 & 39.2 & 44 & 57 & 72.3 \\
\hline
\end{tabular}

fyornm: UNCTAD B2C E-commerce Index 2016

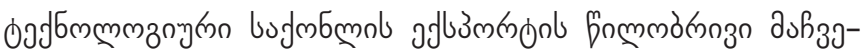

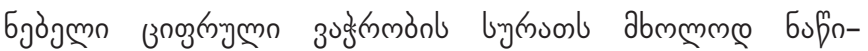

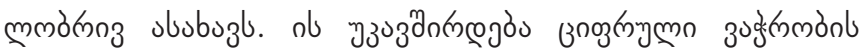

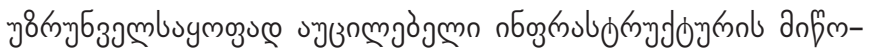

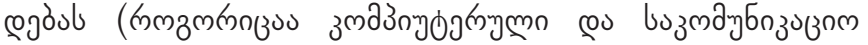

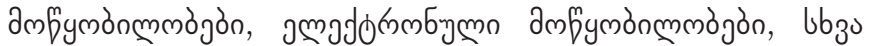

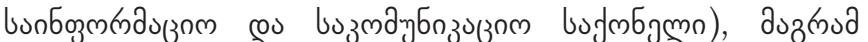

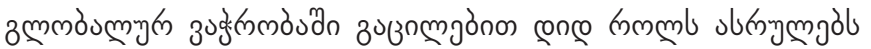

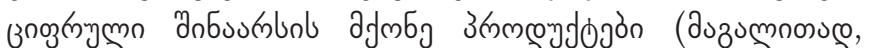

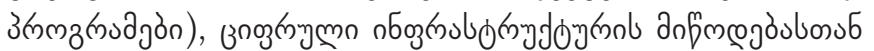

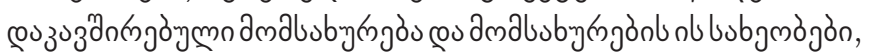

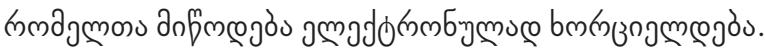

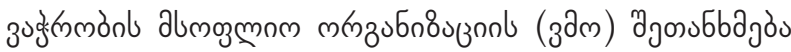

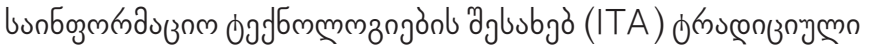

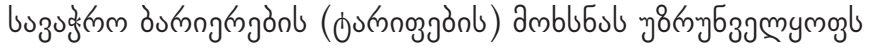

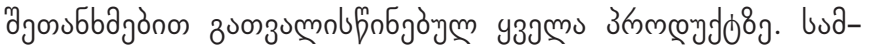

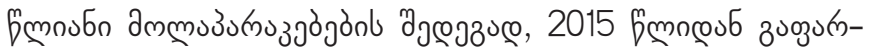

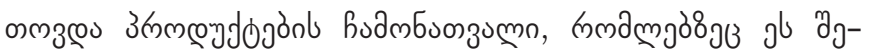

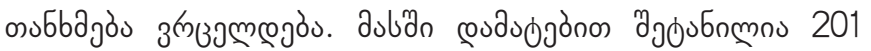

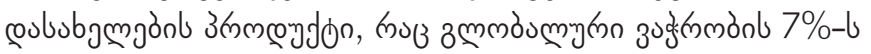

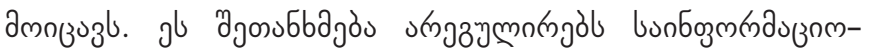

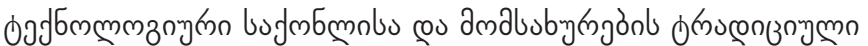

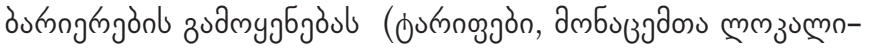

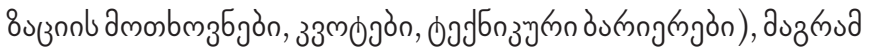

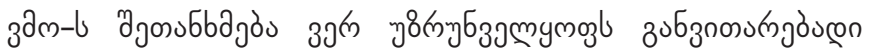

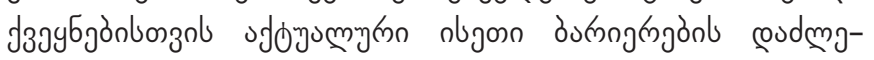

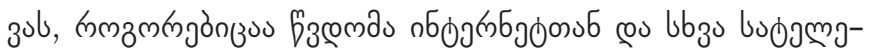

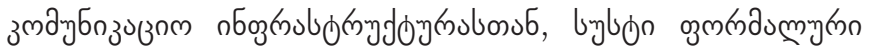

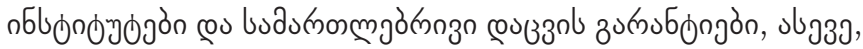

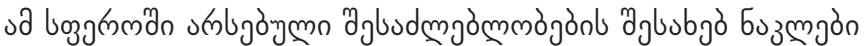

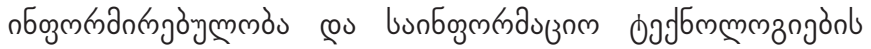

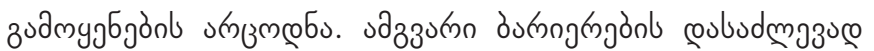

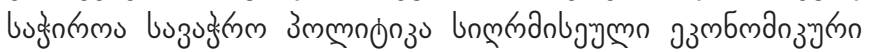

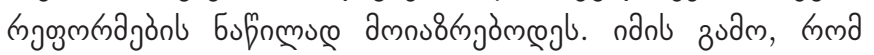

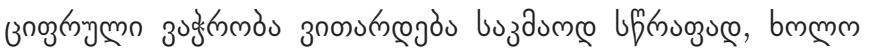
3

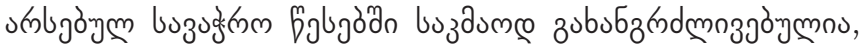

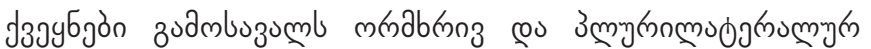

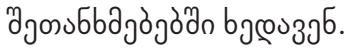

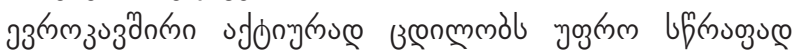

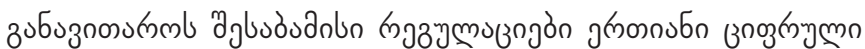

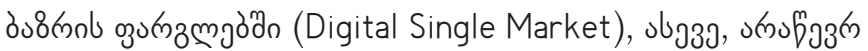

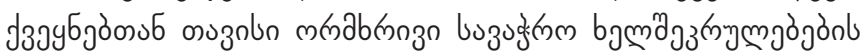
buozyd $39 \mathrm{sm} 8 \mathrm{y}$.

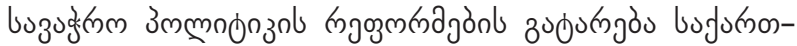

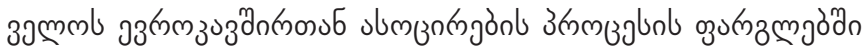

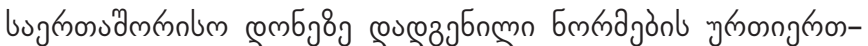

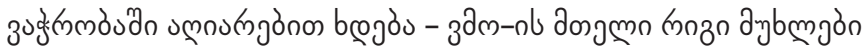

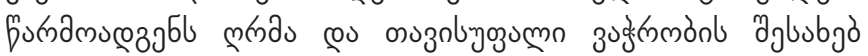

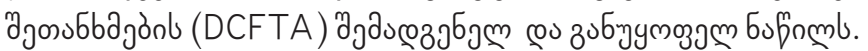

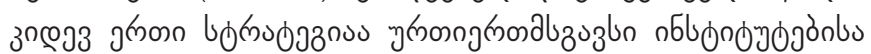

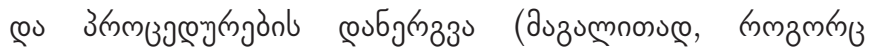

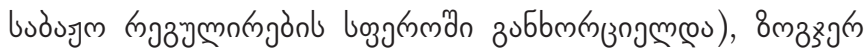

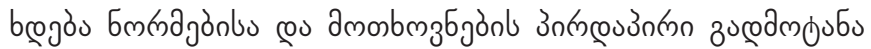

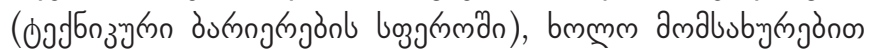

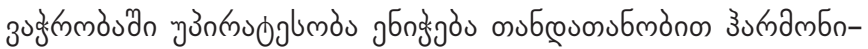

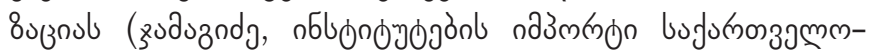

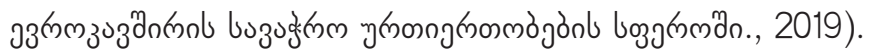

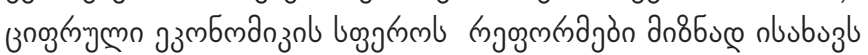

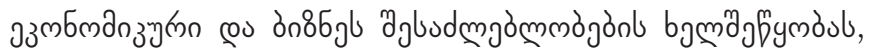

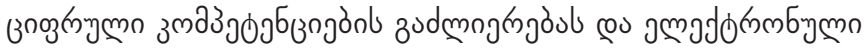

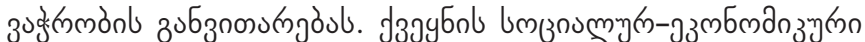

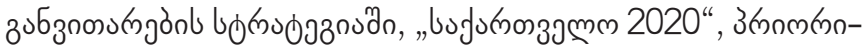

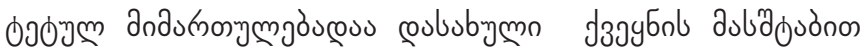

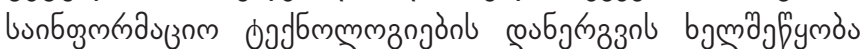

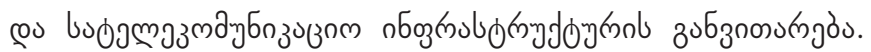

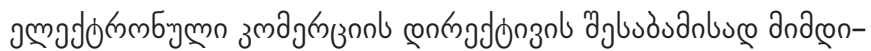

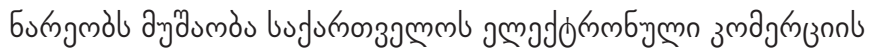

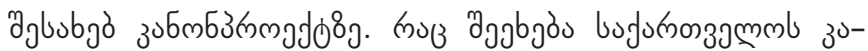

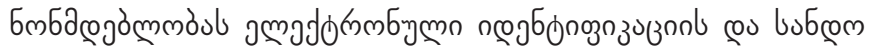

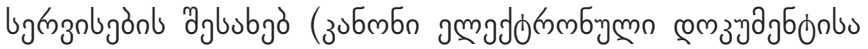

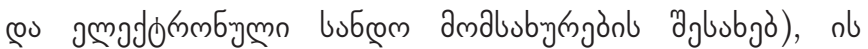

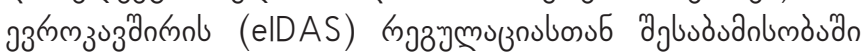
2018 fjgmb amznœu (European..., 2019).

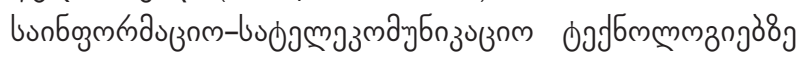

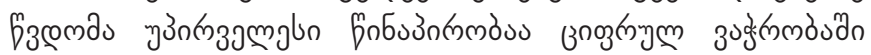

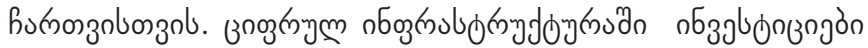

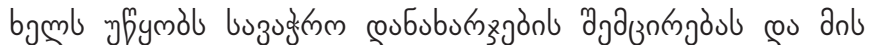

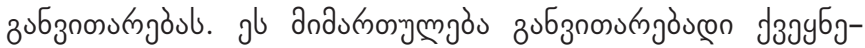

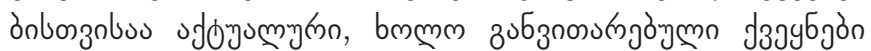




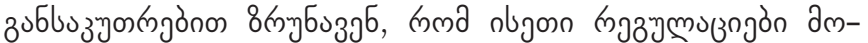

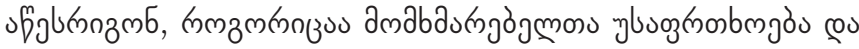
ambuszans cosizo.

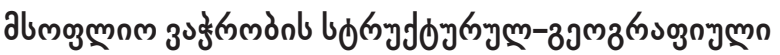 उзмпмпз}

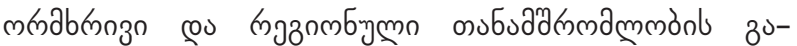

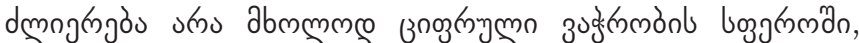

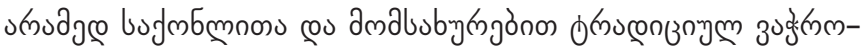

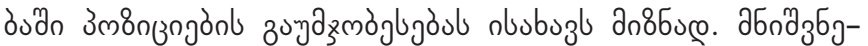

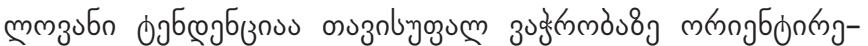

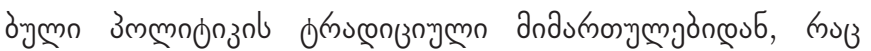

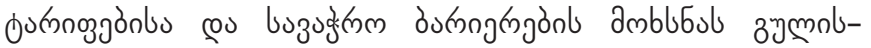

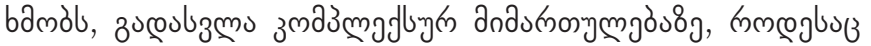

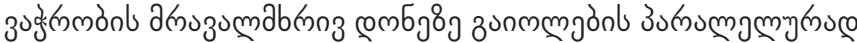

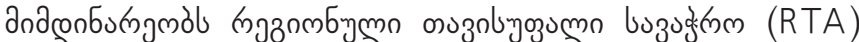

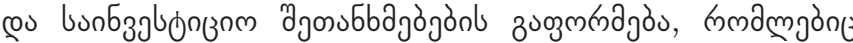

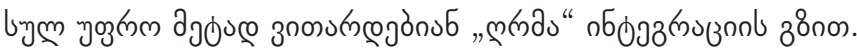

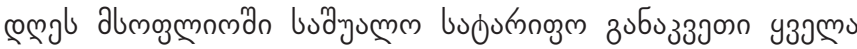

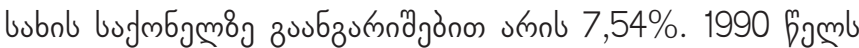

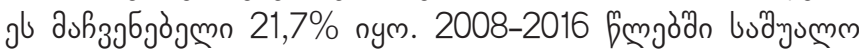

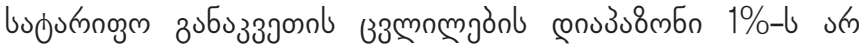

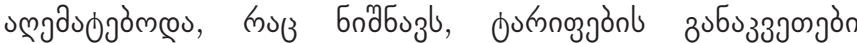

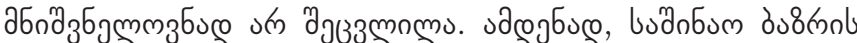

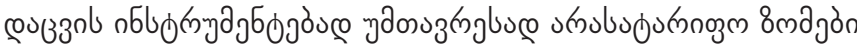

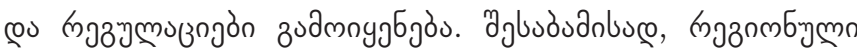

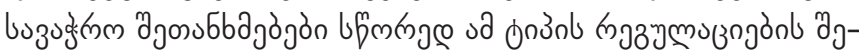
bybojobl sbejб

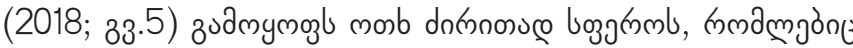

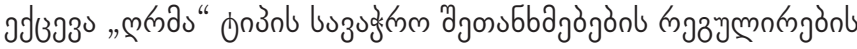

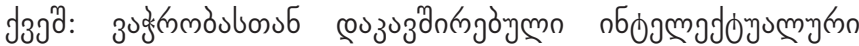

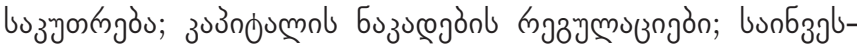

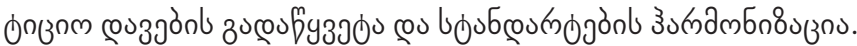

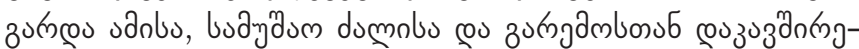

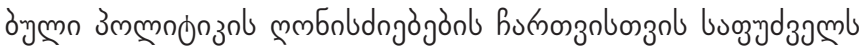

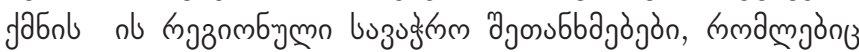

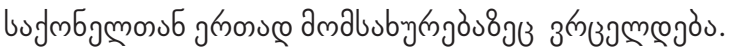

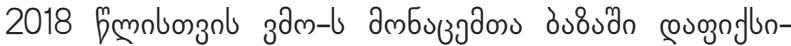

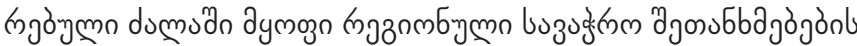

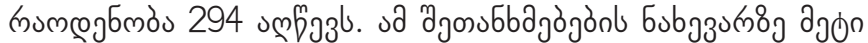

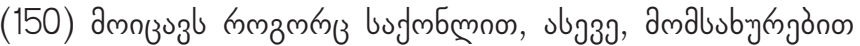

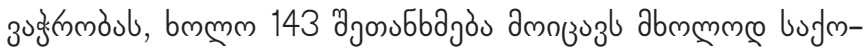

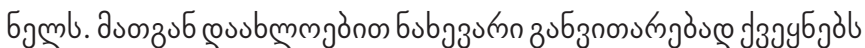

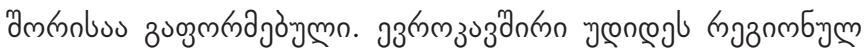

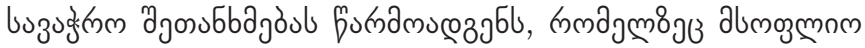

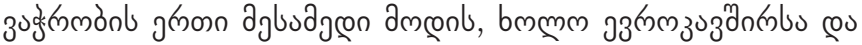

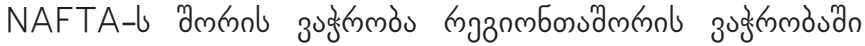

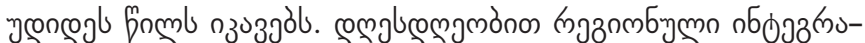

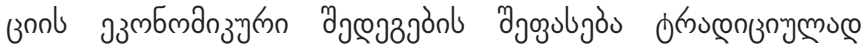

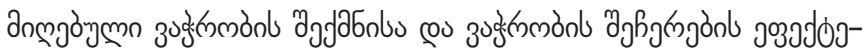

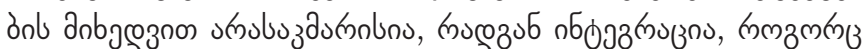

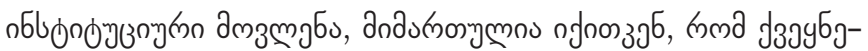

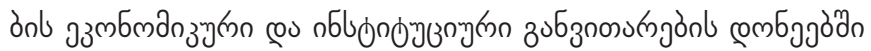
zublubzuzjòjòn unamnogbz mul (Jamagidze, 2010).

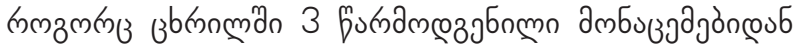

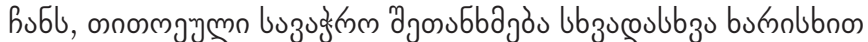

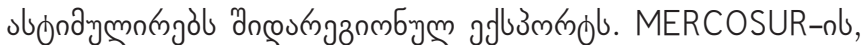

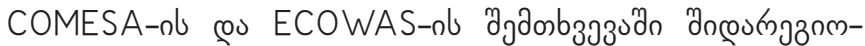

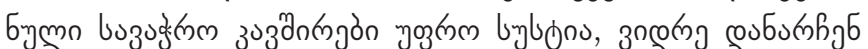

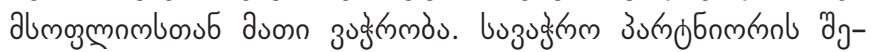

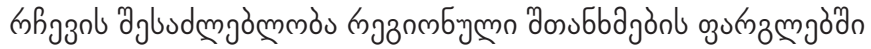

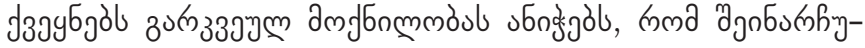

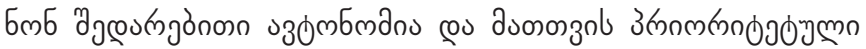

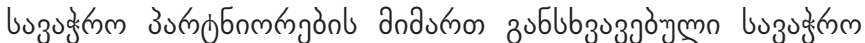

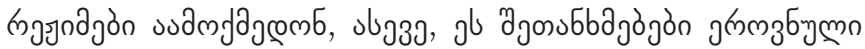

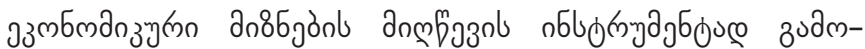

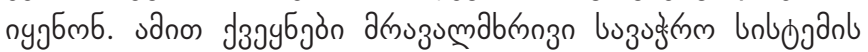

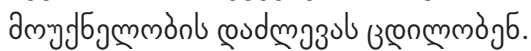

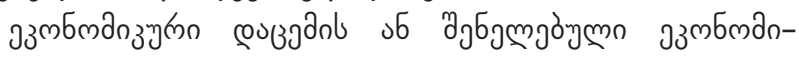

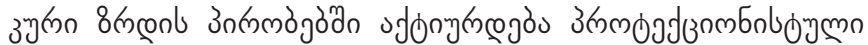

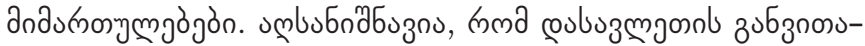

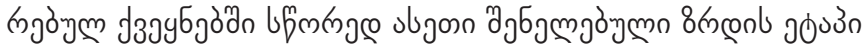

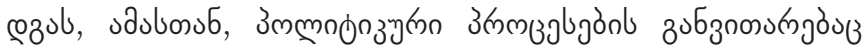

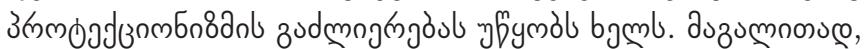

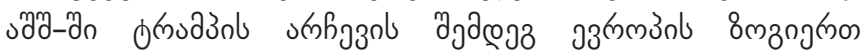

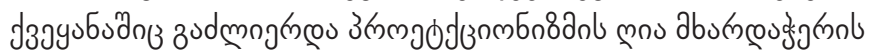
bummso (Rising Protectionism, Challenges, threats and Opportunities for Australia, 2017). उmmojJ(3nmbn8dn g(mon-

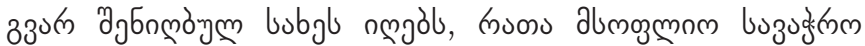

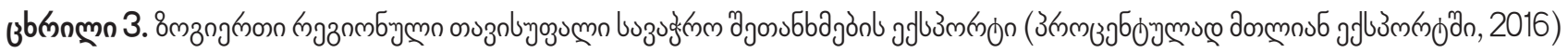

\begin{tabular}{|c|c|c|c|c|c|c|}
\hline & $\mathrm{EU}$ & NAFTA & ASEAN & MERCOSUR & COMESA & ECOWAS \\
\hline 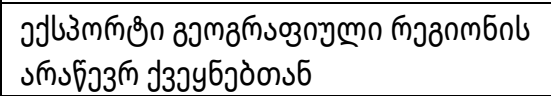 & 6 & - & 43 & 8 & 11 & 14 \\
\hline 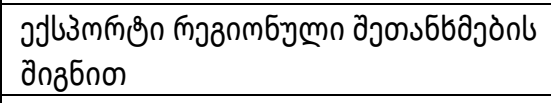 & 64 & 50 & 24 & 13 & 10 & 10 \\
\hline 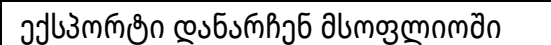 & 30 & 50 & 33 & 79 & 79 & 78 \\
\hline
\end{tabular}

fyornm: World Trade Statistical Review, WTO, 2018, p.74 


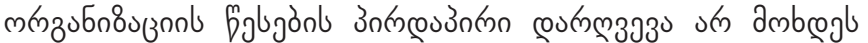

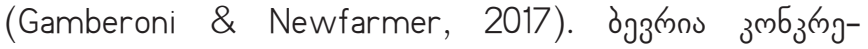

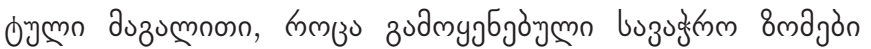

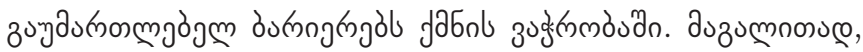

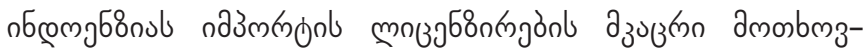

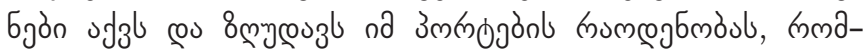

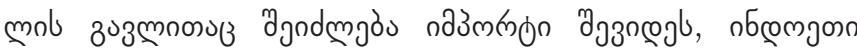

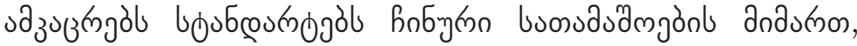

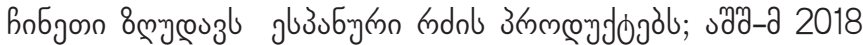

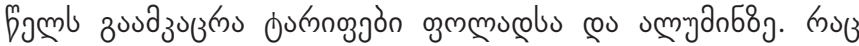

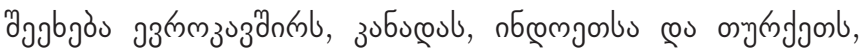

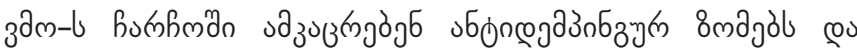

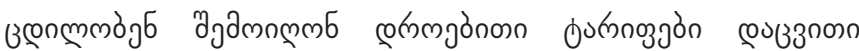

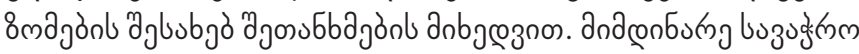

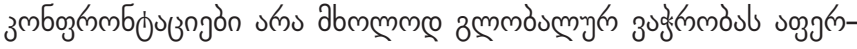

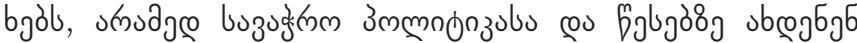

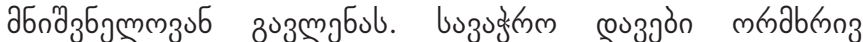

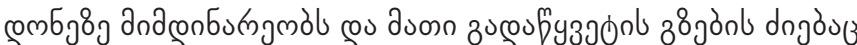

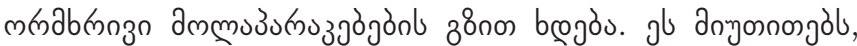

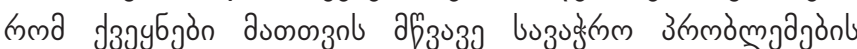

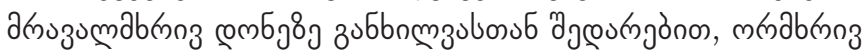

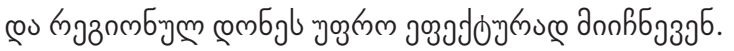

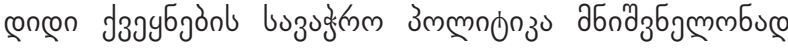

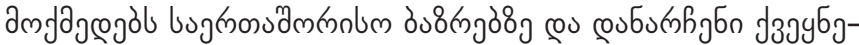

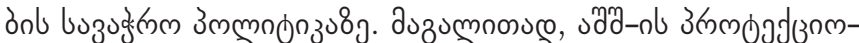

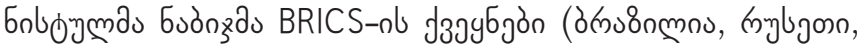

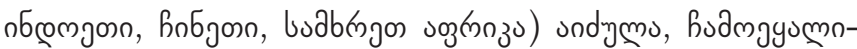

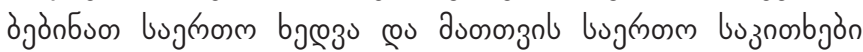

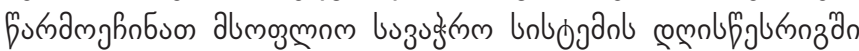

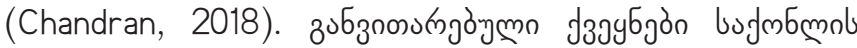

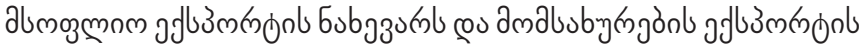

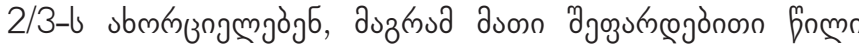

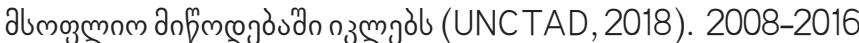

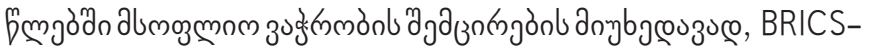

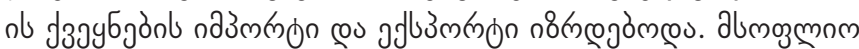

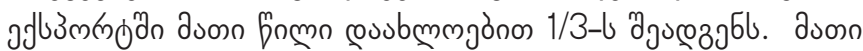

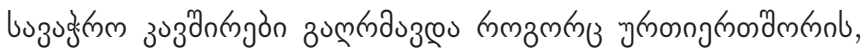

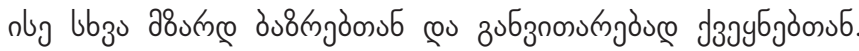

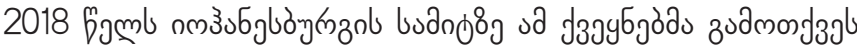

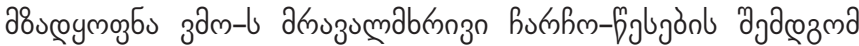

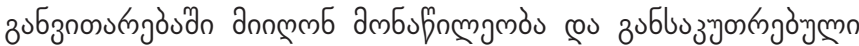

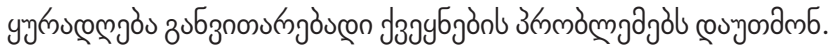

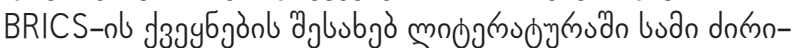

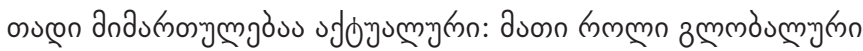

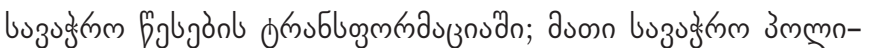

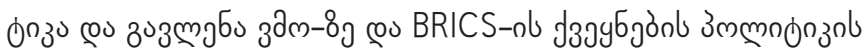

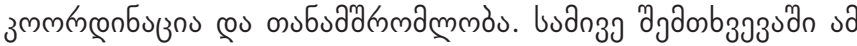

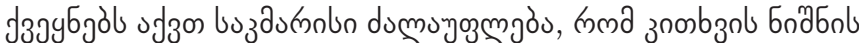

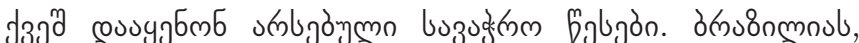

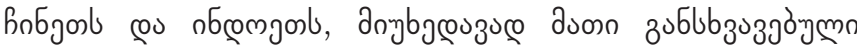

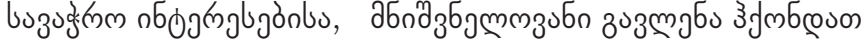

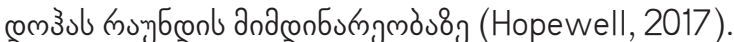

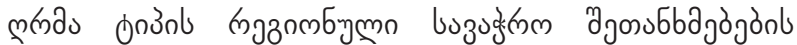
zuofongr

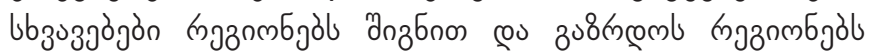

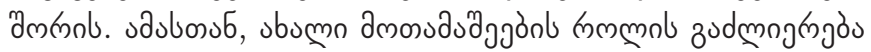

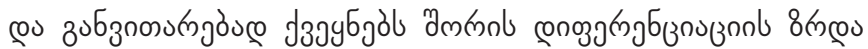

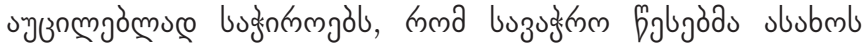
3mलdu

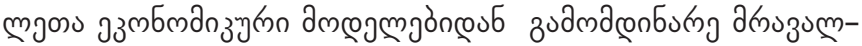
өु

\section{esu336s}

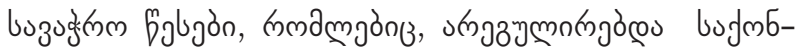

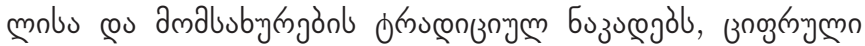

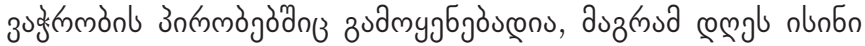

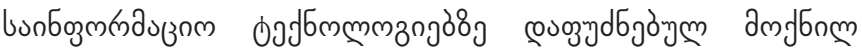

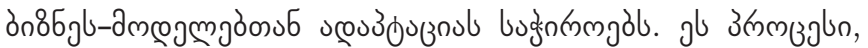

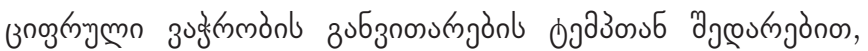
Egma dnaconбurngmall.

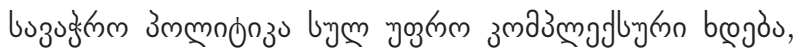

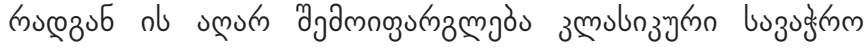

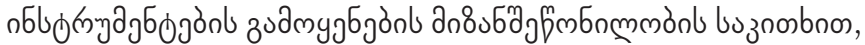

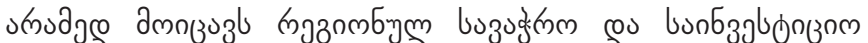

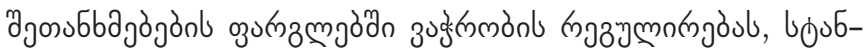

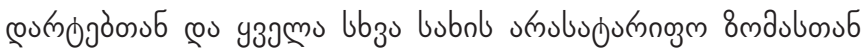

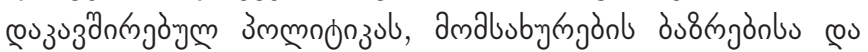

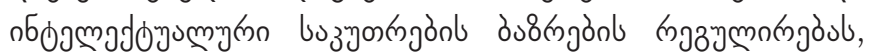
ạz

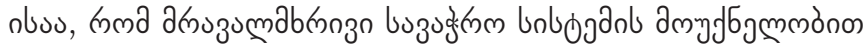

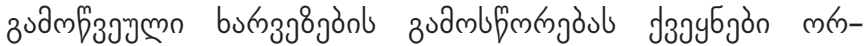

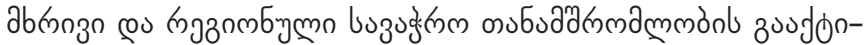

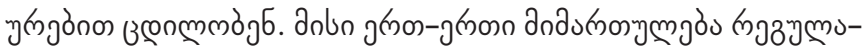

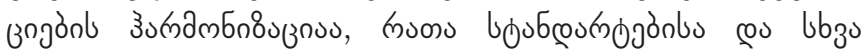

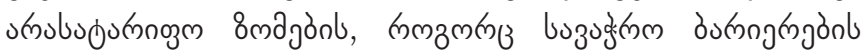

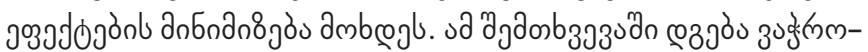

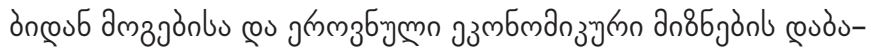

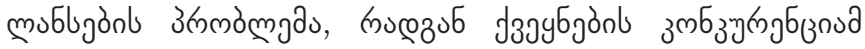

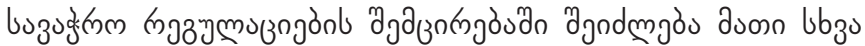

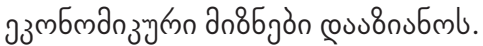

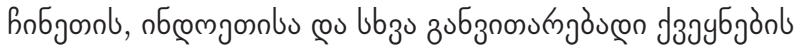

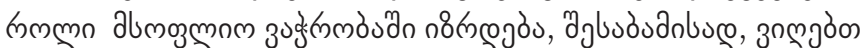

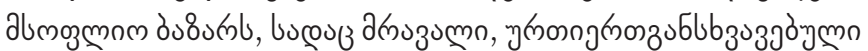

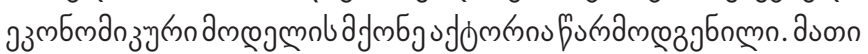

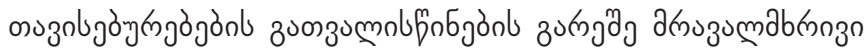

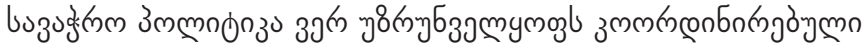

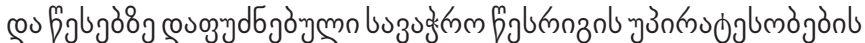

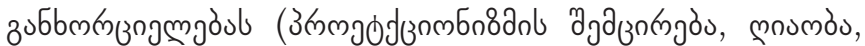

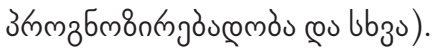




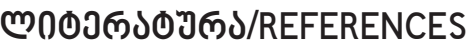

European Commission. (2019). Association Implementation Report on Georgia. https://eeas.europa.eu/sites/eeas/files/2019_ association_implementation_report_georgia_.pdf

Jamagidze, L. (2010). Transnacionaluri korporaciebis roli msoflio ekonomikis globalizaciis processhi. Tbilisi, Universali (in Georgian)

Chandran, Nyshka. (2018). The World's Major Emerging Economies Could End up Benefiting from Global Trade Tensions. https://www.cnbc.com/2018/07/25/brics-could-benefit-from-global-trade-tensions.html

Gamberoni, E., Newfarmer, R. (2017). Trade protection: Incipient but Worrisome Trends, The Collapse of Global Trade, Murky Protectionism, and the Crisis: Recommendations for the G20, Edited by R. Baldwin and Evenett

Goldberg, P. K. (2019). The Future of Trade. Policy can play a role in shaping the future of the ailing multilateral trade system. https://www.imf.org/external/pubs/ft/fandd/2019/06/pdf/the-future-of-global-trade-goldberg.pdf

Hopewell, Kristen. (2017). The BRICS-Merely a Fable? Emerging Power Alliances in Global Trade Governance. International Affairs 93(6):1377-96

International Trade Centre (2017). BRICS countries: Emerging players in global services trade. ITC, Geneva. Productivity Commision. (2017). Rising Protectionism, Challenges, threats and Opportunities for Australia, Research Paper

Rodrik, Dani. (2018). What Do Trade Agreements Really Do? Working Paper 24344 http://www.nber.org/papers/w24344

UNCTAD, (2015). Technical Notes on ICT for Development, No. 7. Geneva.http://unctad.org/en/PublicationsLibrary/tn_ unctad_ict4d07_en.pdf

United Nations Conference on Trade and Development (UNCTAD) (2017): Information Economy Report: Digitalization, Trade and Development.

UNCTAD, (2016). B2C E-commerce Index.

UNCTAD. (2018), Key statistics and Trends in International Trade

World Bank. (2019). World Development Indicators

WTO. (2018). World Trade Report 2018

WTO. (2018). World Trade Statistical Review 